\title{
Ansiedad ante el desempeño musical en estudiantes de música peruanos: diferencias de acuerdo con el género, la institución educativa y el género musical
}

Álvaro M. Chang

Universidad de Lima

\section{Recibido: 17 de marzo del 2016 / Aprobado: 20 de junio del 2016}

Se compararon los niveles de ansiedad ante el desempeño musical (ADM) en una muestra de 455 estudiantes de música peruanos de acuerdo con el género (hombres = 337 , mujeres $=113$, perdidos $=5$ ), la institución educativa (dos privadas y una estatal) y el género musical de especialidad. Solo el género tuvo un efecto en los puntajes de ADM, que mostró mayores niveles en el grupo de mujeres, aunque diversas variables culturales podrían dar cuenta de esta diferencia. No hubo efectos por la institución educativa o el género musical de especialidad en los puntajes de ADM.

ansiedad ante el desempeño musical /género / institución educativa / género musical

\section{Music Performance Anxiety in Peruvian Music Students: Differences According to Gender, Educational Institution and Musical Genre}

Levels of music performance anxiety (MPA) were compared in a sample of 455 Peruvian music students according to gender (males=337; females=113; missing=5), musical institution (two private and one state) and musical genre. Only gender showed an effect on MPA scores, picturing higher scores for the female group, although cultural variables could account for this difference. There were no effects of educational institution or musical genre as determined on MPA scores.

music performance anxiety / gender / educational institution / musical genre

Correo electrónico: alvarochang90@gmail.com 


\section{INTRODUCCIÓN}

La ansiedad ante el desempeño musical (ADM) es un fenómeno experimentado comúnmente por los músicos a lo largo de su vida profesional (Fishbein y Middlestadt, 1988; Kenny, 2011) e implica un sentimiento de ansiedad aprehensiva originada por la interacción de tres factores: vulnerabilidades biológicas, vulnerabilidades psicológicas y experiencias de condicionamiento ansioso o de miedo (Barlow, 2000; Kenny, 2011). Aunque niveles moderados de ADM pueden tener un impacto positivo en el resultado de una presentación (Martínez y Paterna, 2010), niveles más altos pueden generar consecuencias nefastas en el desempeño de los músicos. Estas pueden ir desde disminuir el desempeño realistamente esperado por un músico, pasando por el consumo de sustancias tóxicas, hasta abandonar la profesión (Yoshie, Kudo, y Ohtskuki, 2008). Puede experimentarse independientemente de la edad, nivel de logro profesional, tiempo de práctica, años de entrenamiento y el género musical al que se dedique (Kenny, 2011; Ortiz, 2011; Yoshie et al., 2008).

La ADM presenta componentes fisiológicos, conductuales, cognitivos y afectivos. Fisiológicamente, algunos de los síntomas más comunes son el aumento del ritmo cardiaco, la tasa de sudoración, el aumento en la actividad electromiográfica y una disminución en la temperatura de los dedos (Yoshie et al., 2008). Las manifestaciones conductuales abarcan una aceleración en la actividad muscular o tensión, la cual tiende a aumentar cuando se pasa de una situación de ensayo a una de interpretación frente a un público (Yoshi et al., 2008; Yoshie, Kudo, Murakoshi, y Ohtsuki, 2009). Los pensamientos catastróficos (e. g. "me olvidaré de toda la pieza"), autocrítica (e. g. "mi desempeño estuvo fatal") y una disonancia entre el nivel de desempeño logrado y el que idealmente se tenía (e. g. "sé que puedo tocar mejor que esto") son todos ejemplos del componente cognitivo de la ADM (Deniz, 2007; Kirchner, Bloom, y Skutnick-Henley, 2008; Ortiz, 2011). Finalmente, las manifestaciones afectivas de la ADM incluyen aprehensión, miedo al fracaso y culpa (Kenny, 2011; Kirchner et al., 2008; Ortiz, 2011).

A fin de evitar las consecuencias negativas de los altos niveles de ADM, los músicos pueden adoptar estrategias de afrontamiento poco adaptativas, como consumir drogas ilícitas o no prescritas, tales como alcohol, beta-bloqueadores, marihuana, etc. (Ortiz, 2011; Taylor y Wasley, 2004; West, 2004). Puesto que los estudiantes de música se ven expuestos a distintas situaciones de alto estrés (p. ej. evaluaciones con jurados, recitales y conciertos), es posible que las conductas no adaptativas antes descritas se presenten durante los años de formación.

Durante la última década, se ha incrementado la oferta de educación musical en el Perú, reflejada en nuevas facultades de música y orquestas juveniles. En consecuencia, una mayor cantidad de estudiantes de música deberá afrontar situaciones estresantes que puedan generar 
niveles altos de ADM, junto con las consecuencias negativas que esta conlleva. Puesto que no existía un abordaje psicológico al estudio de la ADM en el Perú, su comprensión se ha basado en investigaciones conducidas en otros contextos. Una de las razones por las cuales no se ha estudiado sistemáticamente este fenómeno en la realidad peruana es por la falta de instrumentos psicológicos diseñados para medir la ADM. Recientemente, el Inventario de Ansiedad ante el Desempeño Musical de Kenny (2009) fue adaptado a una muestra de estudiantes de música peruanos (Chang, 2015a; Chang, 2015b).

Chang (2015a) propuso una estructura factorial en la cual un factor de orden superior subyace y engloba a dos factores de primer orden. Se nombró al factor de orden superior negatividad afectiva ante el desempeño musical y a los factores de primer orden ansiedad ante el desempeño musical y componente depresivo. Esta estructura se basa en el modelo tripartito de la ansiedad y depresión, en el cual ambos fenómenos y su cercana relación son explicados por un factor de orden superior llamado Negatividad Afectiva (Anderson y Hope, 2008; Brown, Chorpita, y Barlow, 1998; Clark y Watson, 1991). De este modo, se corrobora que la naturaleza de la ADM no se limita a enlistar una serie de preocupaciones propias del contexto musical (p. ej. la seguridad ante el escenario, la confianza en la memoria, la seguridad en la interpretación, etc.), sino que su ontología resulta complejizada por una serie de vulnerabilidades emocionales que facilitan la presencia de niveles desorganizadores de ADM (Kenny, 2011).
Así, el objetivo de este artículo es explorar las diferencias en los niveles de ADM obtenidos de una muestra de estudiantes de música peruanos. Particularmente, se compararán los niveles de ADM de acuerdo al género, la institución educativa y el género musical de especialidad. La primera hipótesis afirma que existen diferencias en los niveles de ADM de acuerdo con el género, puesto que esta variable ha demostrado estar asociada con diferencias en los niveles de ansiedad presentados en otros contextos (Barlow, 2000; Kerr-Correa, Zamudio, Hiroce, y Marcassa, 2007). La segunda hipótesis afirma que no existen diferencias en los niveles de ADM de acuerdo con la institución educativa. Finalmente, la tercera hipótesis afirma que no existen diferencias en los niveles de ADM de acuerdo con el género musical de especialidad.

\section{Método}

\section{Participantes}

En Lima Metropolitana, existen alrededor de 1712 estudiantes de música distribuidos en cuatro instituciones privadas $(79,38 \%)$ y dos estatales $(20,62 \%)$. Por medio de un muestreo no probabilístico y accidental, se reunió a 455 estudiantes de música de dos instituciones privadas y una nacional cuya especialidad era Instrumental o Canto. Musicología, Educación Musical, Composición o Producción Musical no fueron contempladas en este estudio. El tamaño de la muestra se estableció de acuerdo con el criterio de Nunnally de 10 participantes por cada ítem del inventario 
(1987). Hubo 337 varones (74,1 \%), 113 mujeres (113\%) y cinco valores perdidos $(1,1 \%)$. La edad promedio fue de 21,19 años $(D E=3.13$, mínimo $=18$, máximo $=40)$. Se contó con 230 estudiantes $(50,54 \%)$ de la Institución Privada A, 84 $(18,46 \%)$ de la Institución Privada B y $141(30,99 \%)$ de la Institución Estatal. Las tres instituciones presentan perfiles de formación musical diferenciados: la Institución Privada A se orienta hacia los géneros contemporáneos o modernos; la Institución Privada B ofrece tanto una formación contemporánea como clásica; y la Institución Estatal, exclusivamente clásica. Los participantes se especializaban básicamente en tres géneros: 283 en estilo moderno (62,2\%), 161 en clásico $(35,4 \%)$ y 10 en folklórico (2,2\%). No obstante, el grupo folclórico fue omitido del presente estudio, puesto que era una cantidad marginal de participantes.

\section{Definición de variables y recolección de datos}

Ansiedad ante el Desempeño Musical: la ADM fue medida con la versión adaptada al español del IADM-K (Chang, 2015a; Chang, 2015b). El inventario adaptado cuenta con 30 ítems en formato de escala Likert con siete opciones de respuesta, que van desde el 0 (Totalmente en desacuerdo) hasta el 6 (Totalmente de acuerdo).

Chang (2015a; 2015b) estimó las evidencias de validez vinculadas a la estructura interna por medio de un análisis factorial exploratorio de segundo orden con la solución Schmid-Leiman (Wolff y Preising, 2005). El análisis reveló un factor de segundo orden y dos factores de primer orden. Uno de los factores de primer orden reunió 21 ítems tales como "A menudo me preparo para un concierto con una sensación de temor y desastre inevitable" y "Me preocupa que un mal concierto pueda arruinar mi carrera", mientras que el otro factor de primer orden contuvo nueve ítems tales como "A menudo siento que la vida no tiene mucho que ofrecerme" y "A menudo siento que no valgo como persona".

Además, se estimaron las evidencias de validez vinculadas a la relación con otras variables, correlacionando las puntuaciones del IADM-K junto con el Inventario de Ansiedad Rasgo-Estado (IDARE), (Spielberger y Díaz-Guerrero, 1970) y el Inventario de Ansiedad de Beck (IAB, como se citó en Thornberry, 2011). Se identificaron efectos fuertes entre el IADM-K, el IDARE y el IAB (Chang, 2015a; Chang, 2015b). Finalmente, se reportaron niveles altos de $\alpha$ ordinal para el factor de segundo orden $(\alpha=.97 ; \mathrm{EEM}=4.87)$, el primer factor de primer orden $(\alpha=.93 ; \mathrm{EEM}=6.11)$ y el segundo factor de primer orden $(\alpha=.92$; $\mathrm{EEM}=3.01$ ).

Institución educativa: se solicitó a los participantes indicar la institución educativa de pertenencia mediante una ficha sociodemográfica.

Género musical de especialidad: los participantes indicaron el género musical de especialidad. También debieron categorizarlo de acuerdo a cuatro opciones: clásico, moderno, folclórico y otros. Tras analizar las respuestas dadas por los 
participantes, se decidió mantener las categorías de clásico y moderno.

\section{Procedimiento}

Se estableció un contacto con las tres instituciones educativas, tras lo cual se asignaron horarios y aulas para la aplicación de los cuestionarios. Todos los participantes leyeron y firmaron un consentimiento informado antes de completar los cuestionarios. En algunos casos, se entrenó a asistentes con un protocolo estándar a fin de agilizar el procedimiento de recolección de datos. Los datos se procesaron con el SPSS versión 21, el Factor versión 9.3.1 (Lorenzo-Seva y Ferrando, 2006) y el GPower versión 3.1.

\section{Análisis estadísticos}

Se emplearon estadísticos paramétricos, pues las pruebas de normalidad sugirieron que las puntuaciones derivadas de la aplicación del IADM-K provienen de una población cuya distribución es normal. Las diferencias en ADM de acuerdo con el género se exploraron por medio de la prueba $t$ de Student para muestras independientes. Las diferencias en ADM de acuerdo con la institución educativa se exploraron con una prueba ANOVA. El valor crítico de $p<.05$ fue utilizado para el contraste de hipótesis. Finalmente, los tamaños del efecto y potencias estadísticas fueron interpretados de acuerdo a Ellis (2010) y Cohen (1992), respectivamente.

\section{Resultados}

\section{Diferencias en $A D M$ de acuerdo al género}

Se ejecutó una prueba de normalidad Shapiro-Wilk en las puntuaciones de ADM de acuerdo con el género. Las puntuaciones obtenidas para los varones $[S-W(337)=0.99, p=.11]$ y para las mujeres $[S-W(113)=0.99, p=.76]$ sugirieron que los puntajes provienen de una población cuya distribución es normal. En la Tabla 1, se resumen estos datos.

\section{Tabla 1}

Media, desviación estándar y prueba Shapiro-Wilk para las puntuaciones de ADM de acuerdo con el género

\begin{tabular}{lcccccc}
\hline \multicolumn{1}{c}{ Género } & $M$ & $D E$ & $n$ & $S-W$ & $g l$ & $p$ \\
Hombre & 67.57 & 27.52 & 337 & 0.99 & 337 & .11 \\
Mujer & 82.53 & 31.14 & 113 & 0.99 & 113 & .76 \\
\hline
\end{tabular}

Nota: $\mathrm{N}=450$. 
En promedio, las participantes obtuvieron puntuaciones más elevadas de ADM $(M=82.53 ; D E=31.14)$ que los varones $(M=67.57 ; D E=27.52)$. Se empleó una prueba $t$ de Student para muestras independientes a fin de determinar si las diferencias eran significativas. La prueba Leven de homogeneidad de varianzas aceptó la hipótesis nula, con lo cual puede afirmarse que no existen evidencias suficientes para asegurar una diferencia en las varianzas de ambas muestras: $F=2.71$, $p=.10$. Asimismo, las diferencias de medias revelaron ser significativas: $t(448)=$ $-4.83, p<.001$. El tamaño del efecto fue mediano y la potencia estadística superó el criterio de Cohen de $.80, d=.50,1-\beta=.81$.

\section{Diferencias en los puntajes de ADM de acuerdo con la institución educativa}

Se llevó a cabo una prueba de normalidad Shapiro-Wilk para las puntuaciones de ADM de acuerdo con la institución educativa de procedencia. En todos los casos, se asumió que las puntuaciones pro- vienen de una población cuya distribución es normal: Institución Privada A, $S$ - $W(230)$ $=0.99, p=.138 ;$ Institución Privada $\mathrm{B}$, $S-W(84)=0.98, p=.39$; e Institución Estatal, $S-W(141)=0.99, p=.30$. La información se resume en la Tabla 2.

La prueba ANOVA de un factor reveló que no existen diferencias significativas en las puntuaciones de ADM de acuerdo con la institución educativa; asimismo, se obtuvo un tamaño del efecto inexistente: $F(2$, 452) $=1.42, p=.24, \eta^{2}=.006,1-\beta=.30$.

\section{Diferencias en los puntajes de ADM de acuerdo al género musical de especialidad}

Se llevó a cabo una prueba de normalidad Shapiro-Wilk para los puntajes de ADM de acuerdo con el género musical de especialidad. Tanto las puntuaciones derivadas de los participantes de estilo clásico $[S-W(161)=0.99, p=.43]$ como las de los de estilo moderno $[S-W(283)=0.99, p=.22]$ presentan evidencias de provenir de una población cuya distribución es normal (ver Tabla 3).

\section{Tabla 2}

Media, desviación estándar y prueba Shapiro-Wilk para las puntuaciones de ADM de acuerdo a la institución educativa

\begin{tabular}{ccccccc}
\hline $\begin{array}{c}\text { Institución } \\
\text { educatival }\end{array}$ & $M$ & $D E$ & $n$ & $S-W$ & $g l$ & $p$ \\
\hline Privada A & 71.34 & 29.31 & 230 & 0.99 & 230 & .03 \\
Privada B & 75.93 & 26.54 & 84 & 0.98 & 84 & .37 \\
Estatal & 69.19 & 30.18 & 141 & 0.99 & 141 & .57 \\
\hline
\end{tabular}

Nota: $\mathrm{N}=455$. 


\section{Tabla 3}

Media, desviación estándar y prueba Shapiro-Wilk para las puntuaciones de ADM de acuerdo con el género musical de especialidad

\begin{tabular}{lcccccc}
\hline $\begin{array}{l}\text { Género musical } \\
\text { de especialidad }\end{array}$ & $M$ & $D E$ & $n$ & $S-W$ & $g l$ & $p$ \\
\hline Clásico & 71.68 & 29.71 & 161 & 0.99 & 161 & .43 \\
Moderno & 71.60 & 29.19 & 283 & 0.99 & 283 & .22 \\
\hline
\end{tabular}

Nota: $\mathrm{N}=444$.

La prueba de Leven corroboró que las varianzas de ambas muestras no se diferencian estadísticamente: $F=0.56, p=.46$. Adicionalmente, a partir de una prueba $t$ de Student para muestras independientes, no se pudo rechazar la hipótesis nula, por lo cual se puede afirmar la no existencia de diferencias estadísticamente significativas en las puntuaciones de ADM de acuerdo con el estilo musical de los participantes: $t(442)=0.03, p=.98, d=.003,1-\beta=.05$.

\section{DISCUSIÓN}

El propósito de esta investigación fue explorar las diferencias en los niveles de ADM en una muestra de estudiantes de música peruanos de acuerdo con el género, la institución educativa y el género musical de especialidad.

Coincidiendo con otros autores (Barlow, 2000; Kerr-Correa et al., 2007; Olatunji y Wolitzky-Taylor, 2009), se registraron diferencias en los niveles de ADM de acuerdo con el género. Particularmente, se detectó un tamaño del efecto mediano en la muestra de participantes mujeres, las cuales superaron a los varones en dicha variable. Algunas explicaciones en torno a estas diferencias son biológicas: la vulnerabilidad a cambios en el estado de ánimo producto de procesos asociados a ciclos de reproducción, tales como el desorden disfórico premenstrual, la depresión postparto y la perimenopausia, se han visto relacionadas con una mayor susceptibilidad en las mujeres para experimentar mayor ansiedad que los varones (Olatunji y Wolitzky-Taylor, 2009). No obstante, estas predisposiciones genéticas pueden reforzarse por factores de crianza y estereotipos de género. Por ejemplo, los estilos de crianza favorecen que los varones se involucren con mayor frecuencia en conductas que fomentan la independencia y la socialización. Esto puede llevar a un mayor desarrollo de la autoeficacia y de estrategias de afrontamiento en comparación con las mujeres, que suelen ser desalentadas o alejadas de involucrarse en estas mismas situaciones (Craske, 2003). Otro factor que podría explicar estas diferencias de género es la inhibición que tanto hombres como mujeres tienen para manifestar sus emociones (Branney y White, 2008; Winkler, Pjrek y Kasper, 2006). De acuerdo con Fine (2010), las pautas de crianza tienden a favorecer que las mujeres 
expresen sus emociones libremente, en tanto que los hombres tenderán a reservarlas como consecuencia de una cultura que sanciona la expresividad emocional en ellos. Al haberse empleado un método de autorreporte para recoger los datos de ADM, estas variables culturales pueden explicar las diferencias aquí presentadas. Futuros estudios que busquen delimitar las diferencias en los niveles de ADM de acuerdo con el género deberán incluir instrumentos de medida más susceptibles a los cambios fisiológicos. En consecuencia, las interpretaciones y decisiones que se sigan a partir de estas diferencias de género deberán tomarse con la mayor de las precauciones y no asumirse como un resultado definitivo.

No se identificaron efectos por pertenecer a una institución educativa o preferir un estilo musical determinado en los niveles de ADM reportados. Puesto que cada institución educativa tiene un perfil musical marcado, es posible considerar a la institución educativa como una medición complementaria del estilo musical de preferencia. De este modo, se concluye que el estilo musical de preferencia no determina ninguna diferencia en los niveles de ansiedad que se puedan experimentar. Estos hallazgos son congruentes con lo planteado por otros autores (Kenny, 2011; Nagel, 2010; Ortiz, 2011; Yoshie et al., 2008) y permiten afirmar que la ADM puede ser experimentada por cualquier músico, independientemente del estilo que cultive. En consecuencia, si no existen efectos del género musical en los puntajes de ADM, todo estudiante de música (cuya especialidad sea Instrumental o Canto) se beneficiaría con programas preventivos o correctivos de ADM. Así, la inclusión de este tipo de intervenciones podría tener consecuencias positivas en los sistemas de educación musical formal, siempre que contemplen tanto los estresores propios del contexto del desempeño musical como las vulnerabilidades emocionales de los virtuales participantes (Chang, 2015a; Chang, 2015b; Ortiz, 2011; Taylor y Wasley, 2004; West, 2004). Contrariamente, una intervención que contemple únicamente las particularidades del contexto musical o solo las vulnerabilidades psicológicas de los participantes no evidenciaría igual calidad en sus resultados. Por ello, futuras investigaciones deberán poner a prueba esta predicción.

A pesar de que los resultados de esta investigación coinciden con los reportados en otros contextos (Fishbein y Middlestadt, 1988; Kenny, 2011; Nagel, 2010; Yoshie et al., 2008), es importante señalar algunas limitaciones del presente estudio. Dadas las limitaciones de financiamiento, no fue posible ejecutar un muestreo probabilístico, lo cual impide generalizar los resultados a otras muestras. Por ello, los resultados aquí reportados deben aplicarse y limitarse a las instituciones y la muestra obtenidas. No obstante, se buscó equilibrar la muestra entre las gestiones privadas y estatales, de tal forma que se pudiese reflejar la distribución poblacional de la manera más fidedigna posible. También se siguió un protocolo de recojo de información estándar, con lo cual algunas variables extrañas pudieron ser controladas. 
A pesar de las limitaciones aquí señaladas, esta investigación es relevante por ser una primera aproximación cuantitativa al estudio de la ADM en la población de estudiantes de música peruanos. Finalmente, se recomienda extender el análisis de las propiedades psicométricas del IADM-K a otras poblaciones de músicos que no fueron incluidas, así como a personas que practican este arte, aunque no hayan sido formadas profesionalmente para ello.

\section{RefERENCIAS}

Anderson, E. R., y Hope, D. A. (2008). A Review of the Tripartite Model for Understanding the Link Between Anxiety and Depression in Youth. Clinical Psychological Review, 28, 275-287. doi: 10.1016/j.epr.2007.05.004

Barlow, D. (2000). Unraveling the Mysteries of Anxiety and its Disorders from the Perspective of Emotion Theory. American Psychologist, 55(11), 1247-1263. doi: 10.1037/0003-066X.55.11.1247

Branney, P., y White, A. (2008). Big Boys don't Cry: Depression and Men. Advances in Psychiatric Treatment, 14(4), 256-262. doi: 10.1192/apt. bp. 106.003467

Brown, T. A., Chorpita, B. F., y Barlow, D. H. (1998). Structural Relationships Among Dimensions of the DSM-IV Anxiety and Mood Disorders and Dimensions of Negative Affect, Positive Affect, and Autonomic Arousal. Journal of Abnormal Psychology, 107(2), 179-192.
Chang, A. M. (2015a). Adaptación y propiedades psicométricas del Inventario de Ansiedad Ante el Desempeño Musical de Kenny (Tesis de licenciatura inédita). Universidad de Lima, Perú.

Chang, A. M. (Agosto, 2015b). Adaptación y propiedades psicométricas del Inventario de Ansiedad Ante el Desempeño Musical de Kenny (IADM-K). Trabajo presentado en la X Conferencia Regional Latinoamericana y III Conferencia Regional Panamericana de Educación Musical, ISME, Lima, Perú. Recuperado de http://congreso.pucp.edu.pe/isme/wp-content/ uploads/sites/8/2013/07/Actas-ISMEPer\%C3\%BA-2015.pdf

Clark, L. A., y Watson, D. (1991). Tripartite Model of Anxiety and Depression: PsychometricEvidence and Taxonomic Implications. Journal of Abnormal Psychology, 100(3), 316-336.

Cohen, J. (1992). A power primer. Psychological Bulletin, 112(1), 155-159. doi: 10.1037/0033-2909.112.1.155

Craske, M. G. (2003). Origins of phobias and Anxiety Disorders: Why More Women than Men? The Netherlands: ELSEVIER.

Deniz, Z. (2007). Performance Anxiety, Dysfunctional Attitudes and Gender in University Music Students. Social Behavior and Personality, 35(10), 1415-1426.

Ellis, P. D. (2010). The Essential Guide to Effect Sizes: Statistical Power, Meta-Analysis, and the Interpretation 
of Research Results. UK: Cambridge University Press.

Field, A. (2009). Discovering Statistics Using SPSS (and Sex and Drugs and Rock 'n' Roll) (3. a ed.). Dubay: Sage.

Fine, C. (2010). Delusions of Gender. USA: W. W. Norton \& Company.

Fishbein, M., y Middlestadt, S. (1988). Medical Problems Among ICSOM: Overview of a National Survey. Medical Problems of Performing Arts, 3(1), 1-8.

Kenny, D. T. (2011). The Psychology of Music Performance Anxiety. New York: Oxford University Press.

Kenny, D. T. (Diciembre, 2009). The Factor Structure of the Revised Kenny Music Performance Anxiety Inventory. Investigación presentada en el Simposio Internacional de Ciencia de la Actuación, Auckland, Nueva Zealanda. Recuperado de http://www.legacy web.rcm.ac.uk/cache/fl0019647.pdf

Kerr-Correa, F., Zamudio, T., Hiroce, V., y Marcassa, A. (2007). Patterns of Alcohol Use Between Genders: A Cross-Cultural Evaluation. Journal of Affective Disorders, 102(1-3), 265275. doi: http://dx.doi.org/10.1016/j. jad.2006.09.031

Kirchner, J., Bloom, A., y SkutnickHenley, P. (2008). The Relationship Between Performance Anxiety and Flow. Medical Problems of Performing Artists, 23, 59-65.

Lorenzo-Seva, U., y Ferrando, P. J. (2006). FACTOR: A Computer Program to Fit the Exploratory Factor Analysis
Model. Behavior Research Methods, 38(1), 88-91. doi: 10.3758/BF03192753

Martínez, M. C., y Paterna, C. (2010). Manual de psicología de los grupos. Madrid: Síntesis.

Nunnally, J. C. (1987). Teoría psicométrica. México D.F.: Trillas.

Olatunji, B. O., y Wolitzky-Taylor, K. B. (2009). Anxiety Sensitivity and the Anxiety Disorders: A Meta-Analytic Review and Synthesis. Psychological Bulletin, 135(6), 974-999. doi: 10.1037/a0017428

Ortiz, A. (2011). Music Performance Anxiety-Part 1: A Review of Its Epidemiology. Medical Problems of Performing Artists, 26(2), 102-105.

Spielberger, Ch., y Díaz-Guerrero, R. (1970). Inventario de ansiedad: rasgoestado (IDARE). Florida: University of South Florida.

Taylor, A., y Wasley, D. (2004). Physical fitness. En A. Williamon (Ed.), Musical excellence: Strategies and techniques to enhance performance (pp. 163-178). New York: Oxford University Press.

Thornberry, G. L. M. (2011). El rol de las creencias irracionales en la relación entre los eventos activadores, $y$ las consecuencias emocionales y conductuales en estudiantes de dos universidades privadas de Lima (Tesis doctoral inédita). Universidad San Martín de Porres, Perú.

West, R. (2004). Drugs and Musical Performance. En A. Williamon (Ed.). Musical Excellence: Strategies and Techniques to Enhance Performance 
(pp. 271-290). New York: Oxford University Press.

Winkler, D., Pjrek, E., y Kasper, S. (2006). Gender Specific Symptoms of Depression and anger attacks. The Journal of Men's Health and Gender, 3(1), 1924. doi: 10.1016/j.jmhg.2005.05.004

Wolff, H. G., y Preising, K. (2005). Exploring Item and High Order Factor Structure with the Schmid-Leiman Solution: Syntax Codes for SPSS and SAS. Behavior Research Methods, 37(1), 48-58.
Yoshie, M., Kudo, K., y Ohtsuki, T. (2008). Effects of Psychological Stress on State Anxiety, Electromyographic Activity, and Arpeggio Performance in Pianists. Medical Problems of Performing Artists, 23, 120-132.

Yoshie, M., Kudo, K., Murakoshi, T., y Ohtsuki, T. (2009). Music Performance Anxiety in Skilled Pianists: Effects of Social-Evaluative Performance Situation on Subjective, Autonomic, and Electromyographic Reactions. Eperimental Brain Research, 199, 117-126. 
